\title{
Finite Element Implementation of Nonlinear Heat Equation in Enthalpy Formulation using ALBERT(A).
}

\author{
Agah D. Garnadi
}

\begin{abstract}
In this paper we describe an implementation of approximate solution to the enthalpy formulation of the Stefan problem.

We allow the thermal properties to have a space and temperature dependence. The algorithm is not explicit in the time variable and hence the stability condition on the time steps is not too severe.

The main aim of this note is program documentation of solving nonlinear heat equation implemented in ALBERT(A) an adaptive finite element method package.

Keywords: Nonlinear Heat Problem, Stefan Problem, Enthalpy Formulation, Kirchoff Transformation, ALBERT(A)

MSC : Primary 65H10, 65M05;Secondary 35K55, 76S05
\end{abstract}


Nomenclature :

$\begin{array}{ll}\Theta & \text { Temperature } \\ H=H(\Theta) & \text { Enthalpy } \\ \beta(\Theta)=u & \text { 'Generalized' Temperature } \\ \Theta_{r} & \text { Reference Temperature } \\ c(\Theta) & \text { heat capacity (Temperature dependent) } \\ \rho(\Theta) & \text { density (Temperature dependent) } \\ k(\Theta) & \text { heat conductivity (Temperature dependent) } \\ \nu & \text { Normal Vector } \\ \Theta_{m} & \text { melting temperature, exactly dependent on Phase diagram } \\ & \text { which dependent on Temperature }\end{array}$




\section{Introduction}

Many industrial (thermal) process involving change of phases. This problem is well known as Stefan's problem, for example :[2]

1. Crystal Growth

2. Freeze Drying

3. Moisture diffusion

4. Laser Ablation

This report documenting an implementation of Finite Element solver for Stefan's problem in ALBERT, later released as ALBERTA [10], a Finite Element toolbox with hierarchical spatial adaptivity. However, we pose the problem in a general setting for a class of nonlinear heat equation which subsumed Stefan's problem instead. Hence we started with a model posed as a fully non-linear heat equation.

\section{Governing Equations}

Let $\Omega \in R^{2}, T>0, Q:=\Omega \times(0, T)$

We concerned with non-linear heat equation of the following in $\Omega$ :

$$
c(\Theta) \rho(\Theta) \frac{\partial \Theta}{\partial t}=\nabla \bullet(k(\Theta) \nabla \Theta)+f(x, t)
$$

On the boundary $\partial \Omega=\Gamma_{D} \cup \Gamma_{N}, \Gamma_{D} \cap \Gamma_{N}=\emptyset$, we set the following condition on Temperature :

$$
\begin{aligned}
\Theta(x, t) & =\Theta_{D}(x), \text { for } x \in \Gamma_{D} \subset \partial \Omega, & & \text { (Dirichlet condition) } \\
-k(\Theta) \nabla \Theta(x, t) \bullet \nu & =\Theta_{N}(x), \text { for } x \in \Gamma_{N} \subset \partial \Omega, & & \text { (Neumann condition) }
\end{aligned}
$$

Initially, over $\Omega$, we set for Temperature an initial condition :

$$
\Theta(x, 0)=\Theta_{0}(x), \text { for } x \in \Omega
$$

We introduce two transformation of variable to remove temperature dependency in the coefficients as follows : 


$$
\begin{array}{r}
H(\Theta) \quad=\int_{0}^{\Theta} c(\zeta) \rho(\zeta) d \zeta, \quad \text { (Enthalpy) } \\
u=\beta(\Theta)=\int_{0}^{\Theta} k(\xi) d \xi, \quad \text { (Kirchoff Transformation) }
\end{array}
$$

See [12], also[8], for the arguments behind this transformation and some physical interpretation.

Assuming that $\beta$ invertible, then we have the following relation :

$$
\Theta=\Theta(u)=\beta^{-1}(u)
$$

Note this is true for the case of most physical problem, since $k(\zeta)$ is nonnegative real-valued, hence $\beta$ is a monotonic function.

The non-linear heat equation (1) after Kirchoff's transformation in Enthalpy formulation:

$$
\frac{\partial H}{\partial t}=\Delta u+f(x, t)
$$

with corresponding boundary conditions ;

$$
\begin{aligned}
u(x, t) & =\int_{0}^{\Theta_{D}(x, t)} k(\xi) d \xi, \text { for } x \in \Gamma_{D} \\
& =u_{D} \\
\frac{\partial}{\partial \nu} u(x, t) & =\int_{0}^{\frac{\partial}{\partial \nu} \Theta(x, t)} k(\xi) d \xi, \text { for } x \in \Gamma_{N} \\
& =u_{N}
\end{aligned}
$$

and initial conditions :

$$
\begin{gathered}
H(x, 0)=\int_{0}^{\Theta(x, 0)} c(\zeta) \rho(\zeta) d \zeta, \text { for } x \in \Omega \\
=H_{0}
\end{gathered}
$$

\subsection{Stefan Problem}

Here, we are more interested in the case where a substance undergoes a change of phases at the temperature $\Theta=\Theta_{m}$, with temperature dependent specific heat on each respective phases :

$$
c(\Theta)=\left\{\begin{array}{ll}
c_{s}(\Theta), & \Theta<\Theta_{m} \\
c_{l}(\Theta), & \Theta_{m} \leq \Theta
\end{array},\right.
$$


then the enthalpy may be written as:

$$
H(\Theta)= \begin{cases}\int_{0}^{\Theta} c_{s}(\zeta) \rho_{s}(\zeta) d \zeta \\ H\left(\Theta_{m}^{-}\right)+\lambda+\int_{\Theta_{m}}^{\Theta} c_{l}(\zeta) \rho_{l}(\zeta) d \zeta, & \Theta<\Theta_{m} \leq \Theta\end{cases}
$$

where: $H\left(\Theta_{m}^{-}\right)=\lim _{\delta \rightarrow 0^{-}} H\left(\Theta_{m}+\delta\right)$

Due to presence of latent heat, observe that there exist a jump discontinuity in Enthalpy function. In practice, we need to regularize the singularity. A regularization trick was suggested by [6] which adopted by [4] to solve Stefan problem using moving finite element.

The regularized enthalpy is of the form :[4]

$$
H(\Theta)= \begin{cases}\int_{0}^{\Theta} c_{s}(\zeta) \rho_{s}(\zeta) d \zeta+\frac{\lambda}{2} \exp \left(-\frac{\left|\Theta-\Theta_{m}\right|}{\epsilon^{-}}\right) & \Theta<\Theta_{m} \\ H\left(\Theta_{m}^{-}\right)+\frac{\lambda}{2}+\int_{\Theta_{m}}^{\Theta} c_{l}(\zeta) \rho_{l}(\zeta) d \zeta-\frac{\lambda}{2} \exp \left(-\frac{\left|\Theta-\Theta_{m}\right|}{\epsilon^{+}}\right), & \Theta_{m} \leq \Theta\end{cases}
$$

To ensure continuous differentiability of $H(\Theta)$ at $\Theta_{m}$, we requires:

$$
c_{s}\left(\Theta_{m}^{-}\right)-c_{l}\left(\Theta_{m}^{+}\right)=\frac{\lambda}{2}\left(\frac{1}{\varepsilon^{+}}-\frac{1}{\varepsilon^{-}}\right)
$$

In [7], a much more simpler regularization were proposed, the proposed regularization is of the following form:

$$
H(\Theta)=\left(\Theta-\Theta_{m}\right)+\frac{\lambda}{2}\left(1+\frac{\left(\Theta-\Theta_{m}\right)}{\sqrt{\left(\Theta-\Theta_{m}\right)^{2}+\varepsilon}}\right)
$$

Another regularization is via Apparent Heat Capacity (AHC) which used in [3], where the regularization is using linear approximation to smooth the jump. We observe that the earlier two regularization type are more favorable during our computation runs compared to the $\mathrm{AHC}$, since we able to use smaller regularization gap : $\varepsilon=\left(\varepsilon^{+}+\varepsilon^{-}\right)$, which means resolving the interval of discontinuity more accurately at the jump. Hence from now on we might use one of the two earlier type of enthalpy regularization.

\section{Finite Element Discretization}

Let : $[0, T]:=\left[0<t_{1}<t_{2}<\cdots<t_{N}=T\right.$

$$
\tau_{n}=t_{n}-t_{n-1} \quad k=1, \cdots, N
$$


Let $H_{D}^{1}(\Omega)=\left\{u ; u, \frac{\partial u}{\partial x_{i}} \in L^{2}(\Omega),\left.u\right|_{\Gamma_{D}}=0\right\}$

Using implicit Euler scheme in time, the time discrete problem :

$$
\frac{1}{\tau_{n}}\left(H^{n}-H^{n-1}\right)-\Delta \tilde{u}^{n}=f^{n-1}
$$

Write :

$$
u^{n}=\left(\tilde{u}^{n}-\tilde{u}_{D}\right) \quad \in H_{D}^{1}(\Omega)
$$

The weak problem :

$$
\begin{gathered}
F\left(u^{n} ; v\right) \\
=\frac{1}{\tau_{n}} \int_{\Omega} H^{n} v \\
+\quad \int_{\Omega} \nabla u^{n} \nabla v \\
F\left(u^{n} ; v\right)
\end{gathered}
$$

\subsection{Finite Element Implementation in ALBERT}

The implementation of heat equation case that accompanied ALBERT source codes (see section 3.3. in [9]) were modified to solve the Stefan problem. Please consult the corresponding section, with a word of warning that the provided case accompanied the software is slightly different in words with the case described in the Manual [9]. However, logically the description in [9] is still valid.

Here, description of main modification are made. Details on the main() program concerning time/space adaptation is not discussed, please refer to the corresponding section on heat equation case in [9].

\subsubsection{Global Variables}

fe_space: a pointer to the finite element space chosen, it is initiallized on the first call of main()

$\mathrm{u} \_\mathrm{h}$ : a pointer to a DOF vector storing the coefficients of the discrete solution for (dependent) variable $u$ ('Generalized' Heat), it is initialized on the first call of main()

$\mathrm{u}_{-} \mathrm{old}$ : a pointer to a DOF vector storing the coefficients of the discrete solution for previous step $U^{(n)}$, it is initialized on the first call of main() 
$\mathrm{T} \_\mathrm{h}$ : a pointer to a DOF vector storing the coefficients of the discrete solution for (dependent) variable $\Theta$ (Temperature), it is initialized on the first call of main()

T_old: a pointer to a DOF vector storing the coefficients of the discrete solution for previous step $\Theta^{(n)}$, it is initialized on the first call of main()

H_h: a pointer to a DOF vector storing the coefficients of the discrete solution for (dependent) variable $H$ (Enthalpy), it is initialized on the first call of main()

H_old: a pointer to a DOF vector storing the coefficients of the discrete solution for previous step $H^{(n)}$, it is initialized on the first call of tt main()

$\mathrm{f} h \mathrm{~h}$ : a pointer to a DOF vector storing the load vector; it is initiallized on the first call of main()

A_matrix: a pointer to DOF matrix storing the stiffness matrix; it is initiallized on the first call of main()

MassD: a pointer to DOF vector storing the diagonal mass matrix; it is initiallized on the first call of main()

A global pointer to the ADAPT_INSTAT structure is used for access in the build() and estimate() routines.

\subsubsection{Functions for leaf data}

See section 3.3.2 in [9]

\subsubsection{Data of the differential equation}

Data for the Stefan problem are the initial values of the Enthalpy $H_{0}$, right hand side $f$, and boundary values $u_{D}$. Note that, all of these values are dependent on temperature variable, $\Theta(x, y, t)$. When the true solution for the temperature is known, $\Theta(x, y, t)$, hence we will now the exact solutions of Enthalpy $H$ and the Kirchoff-transformed of temperature variable, $u$.

\subsubsection{Time discretization}

The model implementation uses Euler implicit time scheme. Initial data is interpolated on the initial mesh,

$$
\Theta_{0}=I_{0} \theta_{0} ; \quad H_{0}=I_{0} H_{0}
$$


The solution $U^{n+1} \approx u\left(., t_{n+1}\right)$ and $H^{n+1} \approx H\left(., t_{n+1}\right)$ is given by $U^{n+1} \in$ $I_{n+1} g\left(., t_{n+1}\right)+X_{n+1}^{o}$ and $H^{n+1} \in X_{n+1}^{o}$ such that :

$\frac{1}{\tau_{n+1}}\left(H^{n+1}, \Phi\right)+\left(\nabla U^{n+1}, \nabla \Phi\right)=\frac{1}{\tau_{n+1}}\left(H^{n}, \Phi\right)+\left(f\left(., t_{n+1}\right), \Phi\right), \quad \forall \Phi \in X_{n+1}^{o}$

Note that, we don't deal with temperature variable directly during computations. We recover the temperature from Enthalpy or it's Kirchoff transformed variables.

\subsubsection{Initial data interpolation}

In our problems, the initial data required is Enthalpy, $H_{0}$, rather than initial Temperature, hence it requires transfomation from $T_{0}$ to $H_{0}$. Initial data $T_{0}$ is just interpolated on the initial mesh, and $H_{0}$ obtained through function evaluation, thus the solve() entry in adapt_instat->adapt_initial will point to a routine interpol_Data0() and which implements this by the library interpolation routine. No build() routine is needed by the initial mesh adaptation procedure.

\subsubsection{Assembly of the discrete system}

Using matrix notation, the non-linear discrete problem can be written as :

$$
\frac{1}{\tau_{n+1}} M\left(\Phi^{n+1}\right)+A \Phi^{n+1}=\frac{1}{\tau_{n+1}} M\left(\Phi^{n}\right)+F^{n+1}
$$

Here, $M=\left(\Phi_{i}, \Phi_{j}\right)$ (up to Dirichlet boundary nodes) denotes the mass matrix and $A=\left(\nabla \Phi_{i}, \nabla \Phi_{j}\right)$ the stiffness matrix. Following the argument as given in the section (3.3.6) in [9], matrix-vector multiplication on the right hand side is realized through local contributions of each single element during assembly.

Most arguments in the section 3.3.6. of [9] is still valid, with an exception that the global mass matrix is stored as a DOF vector, and the local mass operator is lumped before added to the global mass matrix.

static void assembly(DOF_REAL_VEC $* u_{-} h$, DOF_REAL_VEC $* H \_h$,

DOF_MATRIX $*$ A_matrix, DOF_REAL_VEC $*$ MassD, 


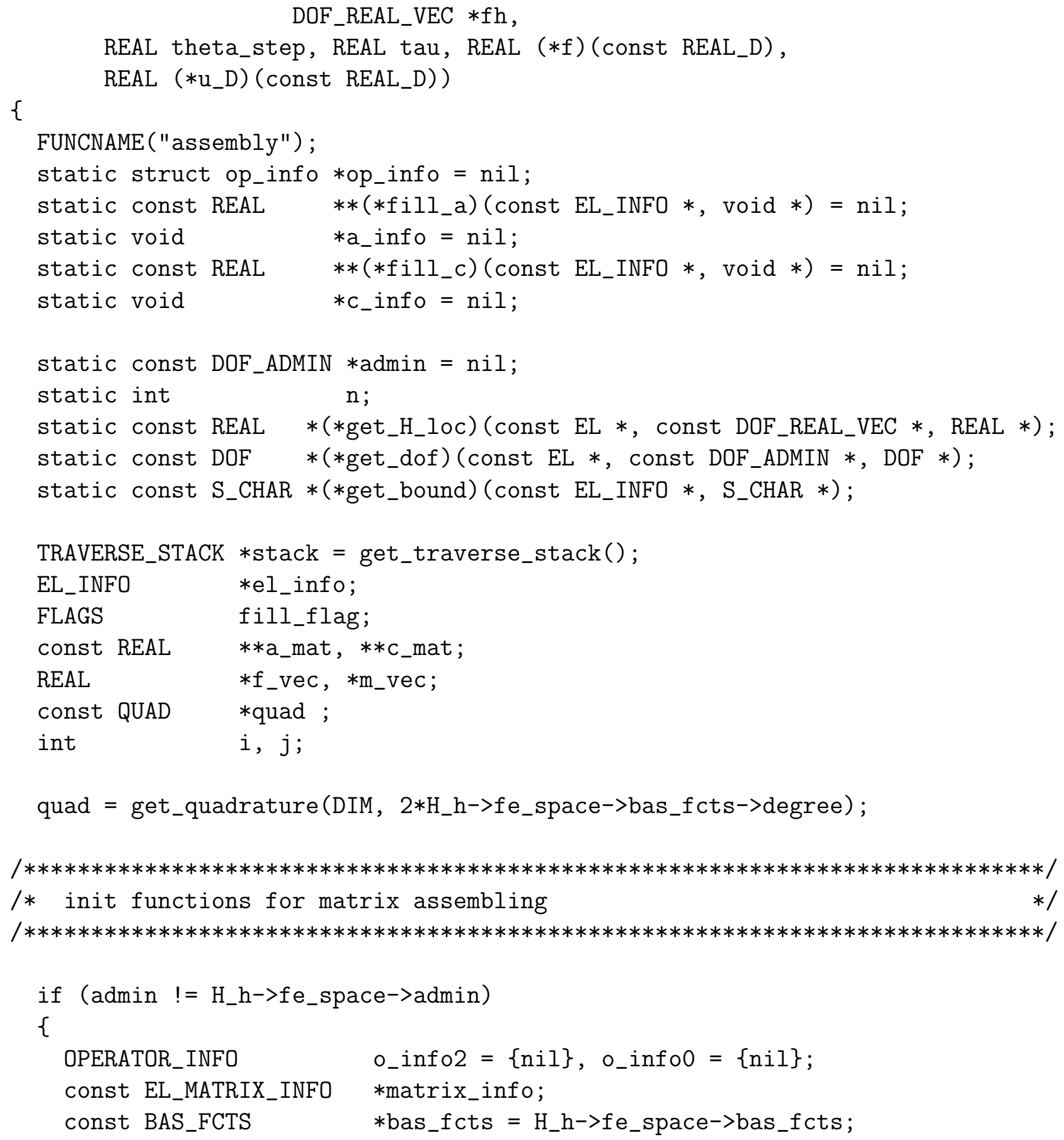




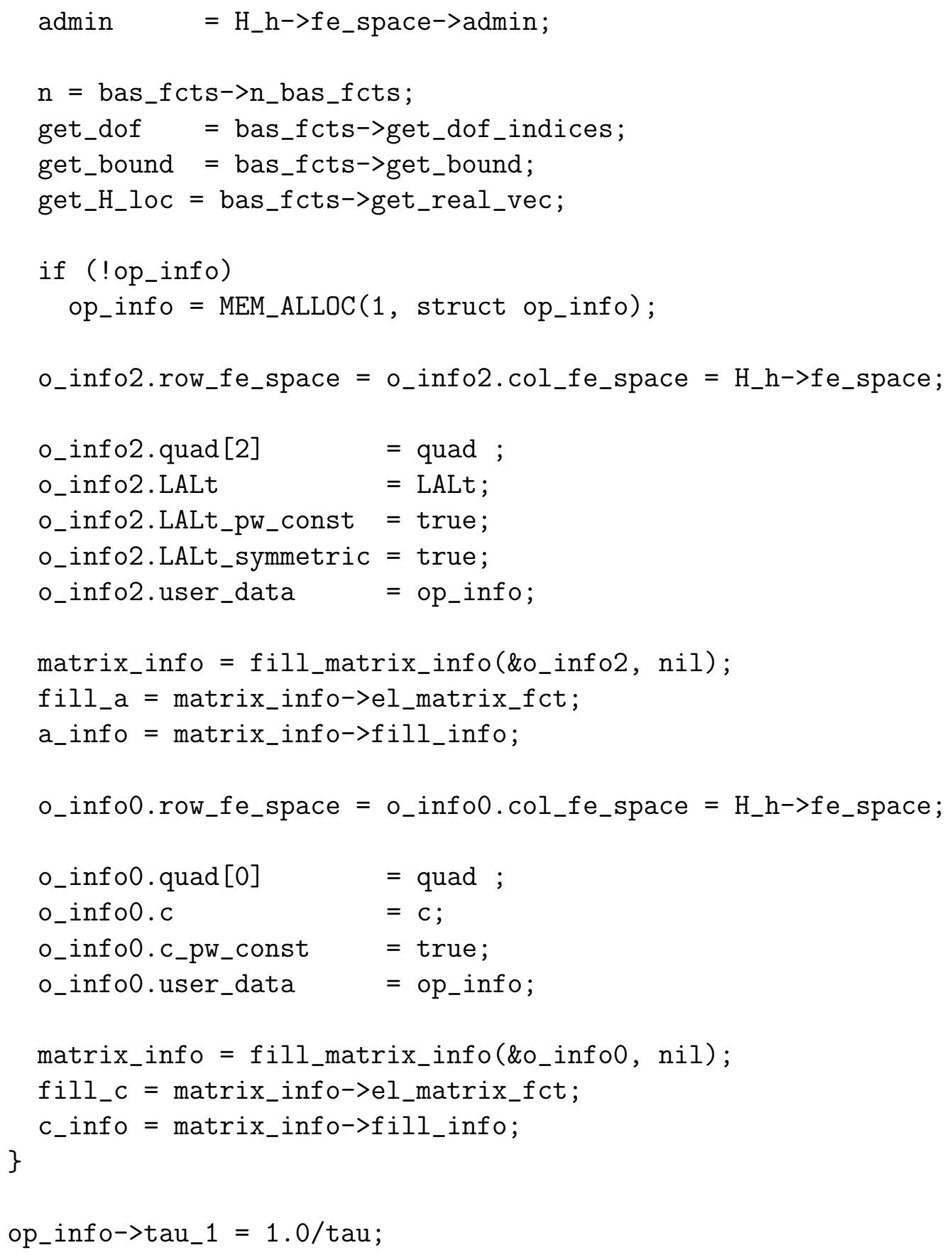


I* and now assemble the matrix and right hand side

clear_dof_matrix(A_matrix);

dof_set (0.0,MassD) ;

$\mathrm{m}_{-} \mathrm{vec}=$ MassD->vec;

dof_set $(0.0, \mathrm{fh})$;

$f_{-} v e c=f h->v e c$;

fill_flag $=$ CALL_LEAF_EL \vert FILL_COORDS \vert FILL_BOUND;

el_info = traverse_first (stack, H_h->fe_space->mesh, -1 , fill_flag);

while (el_info)

\{

const REAL $*$ H_loc $=(*$ get_H_loc $)\left(e l \_i n f o->e l, H \_h\right.$, nil $)$;

const DOF $\quad *$ dof $\quad=$ (*get_dof) (el_info->el, admin, nil);

const S_CHAR $*$ bound $=$ (*get_bound) (el_info, nil);

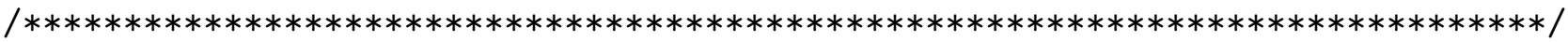

/* initialization of values used by LALt and c

/********************************************************************************/

op_info->det = el_grd_lambda(el_info, op_info->Lambda);

a_mat $=$ fill_a $\left(e l_{-}\right.$info, a_info $)$;

$c_{-}$mat $=f i l l_{-} c\left(e l_{-}\right.$info, $c_{-}$info $)$;

/********************************************************************************/

/* add_to A_matrix $+=$ a (psi_i,psi_j)

$* 1$

$/ * * * * * * * * * * * * * * * * * * * * * * * * * * * * * * * * * * * * * * * * * * * * * * * * * * * * * * * * * * * * * * * * * * * * * * * * * * * * * * * * * * *) /$

add_element_matrix(A_matrix, 1.0, n, n, dof, dof, a_mat, bound);

/********************************************************************************/

/* $f+=1 /$ tau*m $\left(T_{-}\right.$old,psi_i)

/* add_to MassD $\quad+=1 /$ tau*m(psi_i,psi_j)

/********************************************************************************/ 


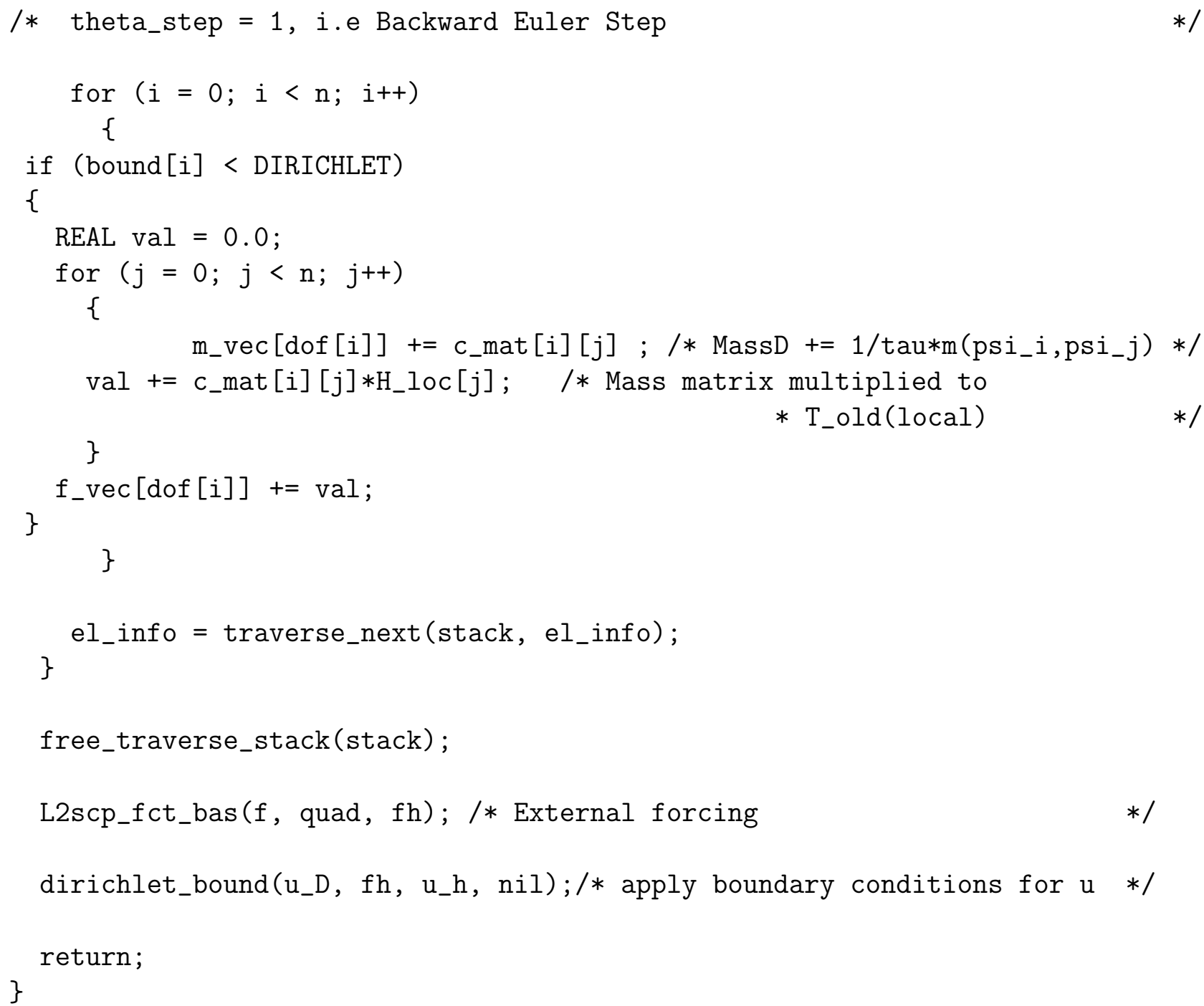

For each time step, the build() just calls assembly() routine described above, with evaluation times for right hand sides and boundary values adjusted to the next step.

static void build(MESH $*$ mesh, U_CHAR flag) \{ 


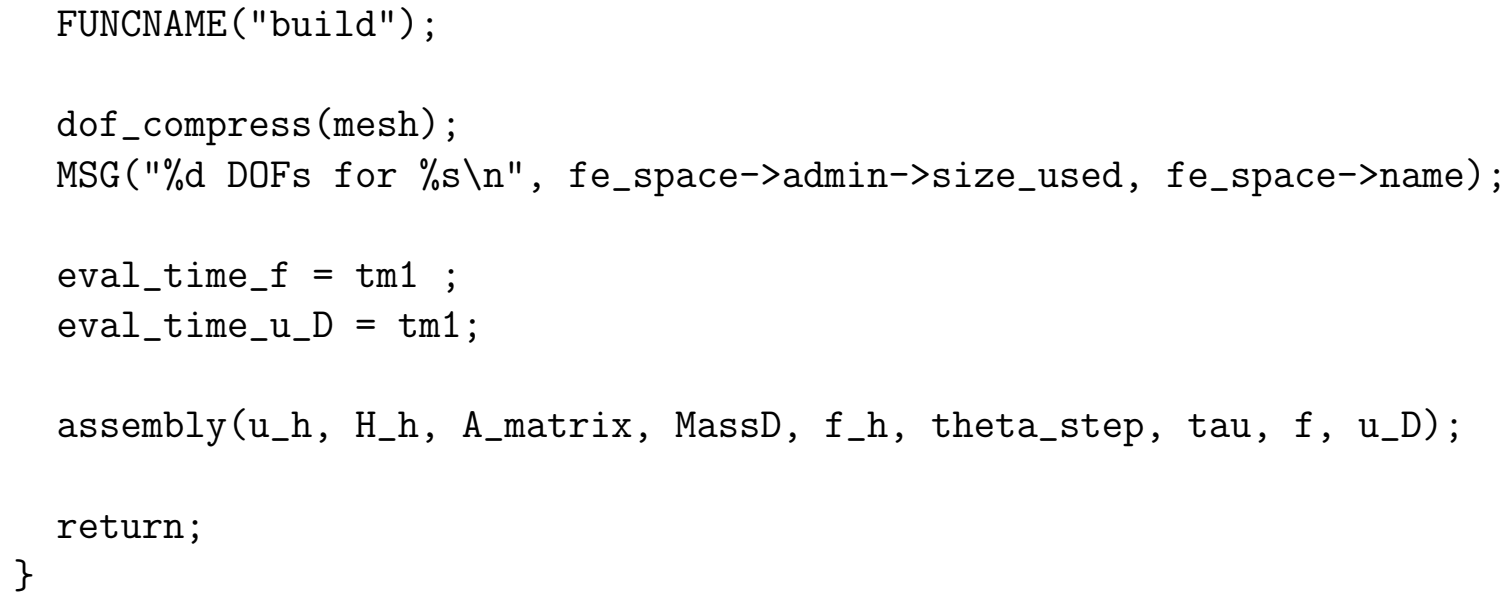

The resulting non-linear systems solved using nlsor() routine. This is done via the solve() routine described in the section 3.1.7. in [9].

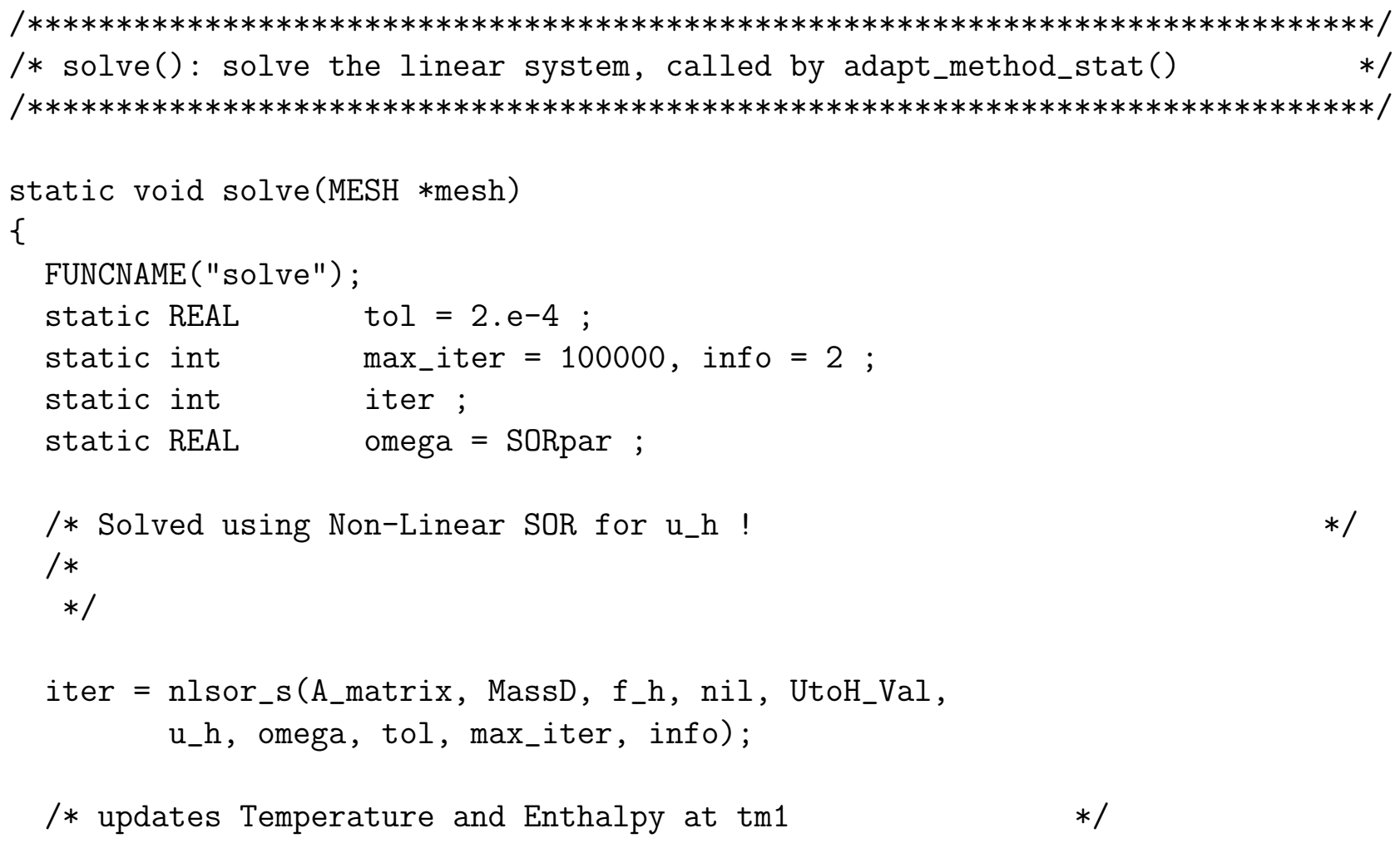




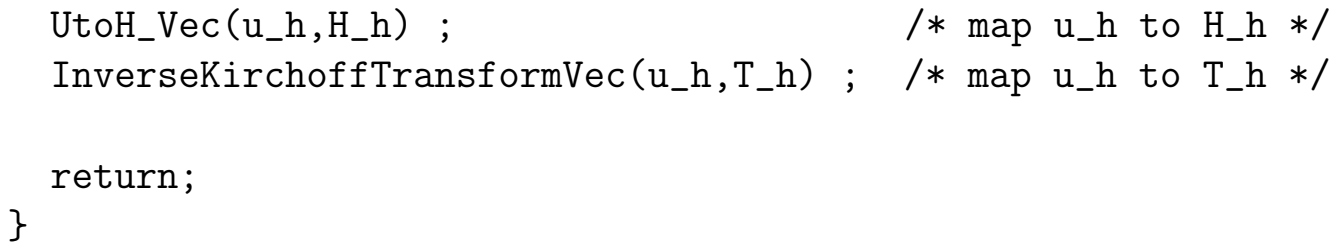

\section{Nonlinear SOR}

To solve the resulting non-linear discrete systems, we take resort using nonlinear SOR[12]. Under several assumptions on $H$ and $u$ as functions of temperature, $T$, we express the discrete non-linear problem into the following form :

$$
M H(U)+A U=b
$$

with requirement : $a_{i i}>0, \quad m_{i i}>0$, and let $r_{i}(\zeta)=m_{i i} h(\zeta)+a_{i i} \zeta$, under an assumption that it is invertible, with inverse function denoted by $r_{i}^{-1}$. Rewriting $M H(U)+A U=b$ into the form :

$$
\begin{aligned}
r_{i}\left(U_{i}\right)=b_{i} & -\sum_{i>j}\left\{a_{i j} U_{j}+m_{i j} H_{j}\left(U_{j}\right)\right\} \\
& -\sum_{i<j}\left\{a_{i j} U_{j}+m_{i j} H_{j}\left(U_{j}\right)\right\}
\end{aligned}
$$

Due to diagonal format of the mass matrix, the nonlinear SOR algorithms proceed as follows[12]. Let $\omega \in[1,2)$ be a provided relaxation parameter.

$$
\begin{aligned}
& \text { Iterates on } k=1, \cdots, \\
& \qquad \begin{aligned}
U_{i}^{k+\frac{1}{2}} & =r_{i}^{-1}\left(b_{i}-\right. \\
U_{i}^{k+1} & \left.=\left(\sum_{i<j} a_{i j} U_{j}^{k+1}+\sum_{i>j} a_{i j} U_{j}^{k}\right\}\right) \\
& =(1-\omega) U_{i}^{k}+\omega U_{i}^{k+\frac{1}{2}}
\end{aligned}
\end{aligned}
$$

until convergence reached.

Clearly, we need a root solver for scalar function $r(\zeta)$ for each iteration. We resolve this via Newton method.[12] The source code can be consulted in the appendix. 


\section{Note}

As mentioned before, at a single iteration sweep, we have to find a root from $r(\zeta)$ for each degree of freedom. In the case the derivative of $r(\zeta)$ is not available, then during root finding iteration in the (scalar)-Newton iteration, finite-difference approximation of $r^{\prime}(\zeta)$ are utilized. In our experience, this situation create a hindrance in some way to arrive to the root for a given tolerance, penalized computational time at each sweep. Hence, availability of analytical derivative of $r(\zeta)$ is quite desirable.

\section{$5 \quad$ Numerical Test}

Let $\Omega=(0,1)^{2}, \Gamma_{L}=\{0\} \times[0,1], \Gamma_{R}=\{1\} \times[0,1], \Gamma_{B}=[0,1] \times\{0\}, \Gamma_{T}=$ $[0,1] \times\{1\}$.

We set parameters for nlsor solver be : $\omega=1.1$, tolerance $=2.0 e-4$, and the sor iteration will be bailed out after $10^{5}$ iterations.

We consider three cases involving phase-change process in $\Omega$ for a material with $\rho_{i}=c_{i}=k_{i}=1.0$. The material possessed a latent heat of value 1,i.e. $\mathrm{L}=1$, with solidification/melting temperature at zero. We apply zero Neumann boundary condition on $\Gamma_{B} \cup \Gamma_{T}$, i.e. insulation at top and bottom boundaries. While at the left boundary, $\Gamma_{L}$, we prescribed a constant temperature at $\left.T\right|_{\Gamma_{L}}=-0.9$, while on the right boundary, $\Gamma_{R}$, we set a fix boundary temperature at $\left.T\right|_{\Gamma_{R}}=0.5$.

The domain $\Omega$ is triangulated using a fixed uniform grid consists of 16, 389 triangles and 8,319 nodes. A constant time step $\Delta t=0.0012$ are taken during the test. And the regularization parameter set at $\epsilon=1.0 e-5$, for regularized enthalpy as suggested by Grosmann and Noack[7].

We consider three prescribed initial values for the problem, where for each initial values, the evolving process shows solidification process, melting, and both process occurs. In the first case, initially the material is in liquid phase at uniform temperature $T_{0}=0.5$, this case shows solidification process towards steady-state solution. In the second case, we set initially at solidification temperature $T_{0}=0$, which the process undergoes solidification and melting at the same time before reaching steady-state temperature. While the third case, we set initially the material is in solid phase at uniform temperature $T_{0}=-0.5$, this case showsmelting process towards steady-state 
solution.

\section{References}

[1] V. Alexiades, G. Amiez, \& P. A. Gremaud, 1996, Supertime-stepping Accelleration of Explicit Schemes for Parabolic Problems, Com. Num. Meth. Eng., 12 (1996), p.31-42

[2] V. Alexiades \& A. D. Solomon, 1993, Mathematical Modeling of Melting and Freezing Processes, Hemisphere Publ. Co., Washington DC, ISBN $1-56032-125-3$

[3] C. Batur, A. Srinivasan, W. M. B. Duval, N. B. Singh, D. Golovaty, 1999, On-line control of solid-liquid interface by state feedback, J. Cryst. Growth 205, p395-409

[4] G. Beckett, J. A. Mackenzie, \& M. L. Robertson, 1999, A Moving Mesh Finite Element Method fo the Solution of Two-Dimensional Stefan Problems, Technical Report n0 99/26, Dept. Math, Uni-Strathclyde. Appear in Journal of Computational Physics, Vol. 168, No. 2, Apr 2001, pp. 500-518

[5] St. Boschert, A. Schmidt, \& K. G. Siebert, 1999, A Numerical Simulation of Crystal Growth by the Vertical Bridgman Method, AlbertLudwigs-Universität Freiburg, Mathematische Fakultät Preprints No 9924

[6] P. W. Egolf \& H. Manz, 1994, Theory and modelling of phase change materials with and without mushy regions, Int. J. Heat Mass Transfer, 37:2917-2924

[7] C. Grossmann \& A. Noack, 2002, Smoothing and Rothe's Method for Stefan Problem in Enthalpy Form, J. Comp. App. Math., V 138(2), 347-366

[8] P. Knabner, Freie Randwertprobleme: Analysis und Numerik, Lecture Notes, IAM I, Universität Erlangen-Nürnberg 
[9] A. Schmidt, \& K. G. Siebert, 2000. ALBERT: An adaptive hierarchical finite element toolbox. Albert-Ludwigs-Univ., Math. Fak..

[10] A. Schmidt and K.G.Siebert: Design of adaptive finite element software: The finite element toolbox ALBERTA, Springer LNCSE Series 42 (2005).

[11] A. A. Wheeler, 1993, Description of Transport Processes, Handbook of Crystal Growth, Vol 1. Elsevier.

[12] R. E. White,1985, An Introduction to the finite element method with applications to nonlinear problems, Wiley, New York 


\section{APPENDIX A. User supplied function.}

To use the main driver, the user should provides geometrical data through Macro file, adaptivity infos through INIT file, and a file contains initial data, boundary conditions, and material parameter as user supplied function. One important function needed by the driver is a scalar-valued functions, taking a value in $u$, and map it to $H$, i.e. the following map :

$$
H(u)=\int_{0}^{\beta^{-1}(u)} \rho(\zeta) c(\zeta) d \zeta
$$

Essentially it is this map that holds material parameter function at once, to be passed to the main driver. In the case analytical expression of $H(u)$ is available, it is recommended to use non-linear solver which accepts function call for $\frac{d}{d u} H(u)$. See commentary about this in the other appendix on nonlinear SOR later.

The following code write up shows an example of implementation for the case of Silicon. In this write-up, we use the regularization given in [4], the user may found it is not difficult to replace the regularization expression for other expression, such as regularization given in [7]. Below, we utilize non-linear solver without calls to $\frac{d}{d u} H(u)$ :

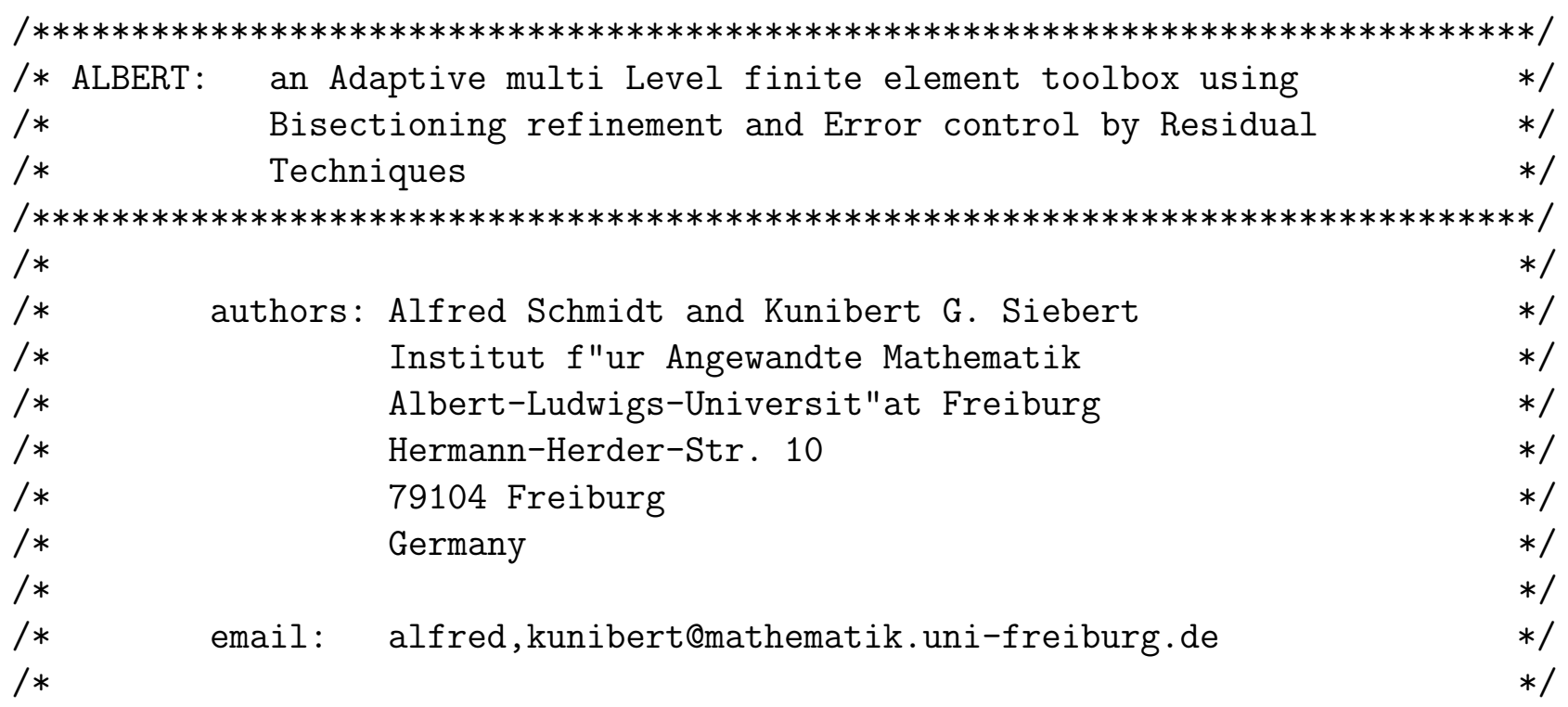


/* (c) by A. Schmidt and K.G. Siebert (1996-99)

/* solver for NonLinear Stefan problem ${ }^{\sim}$ :

/* Irho $c_{-} i \mathrm{~T}, \mathrm{t}-\operatorname{div}\left(\mathrm{k}_{-} \mathrm{i} \operatorname{grad} \mathrm{T}\right)=0, \mathrm{i}=1, \mathrm{~s} * /$

/* using Implicit Euler Scheme

/* The methods used are via Kirchoff Transformation :

/* And Enthalpy functions:

/* $\quad \mathrm{H}(\mathrm{T})=\backslash$ int_0^T $\backslash r h o(\backslash x i) c(\backslash x i) d \backslash x i$

/* such that the original problem turned into:

\#include <albert.h>

\section{/*}

\#define AmplitudoL 20.0

\#define AmplitudoR 10.0

$* 1$

\#define AmplitudoL 1000.0

\#define AmplitudoR 2000.0

\#define T_shift 0.0

\#define T_melting 1685.0

\#define phi_root 0.05254380387956843

\#define epsReg 0.125

\#define Latent 1800000.0 
REAL C_S $=900.0, c_{-} 1=1000.0$;

REAL rho_s $=2490.0$, rho_l $=2490.0$;

REAL k_s $=22.0, k_{-} l=64.0$;

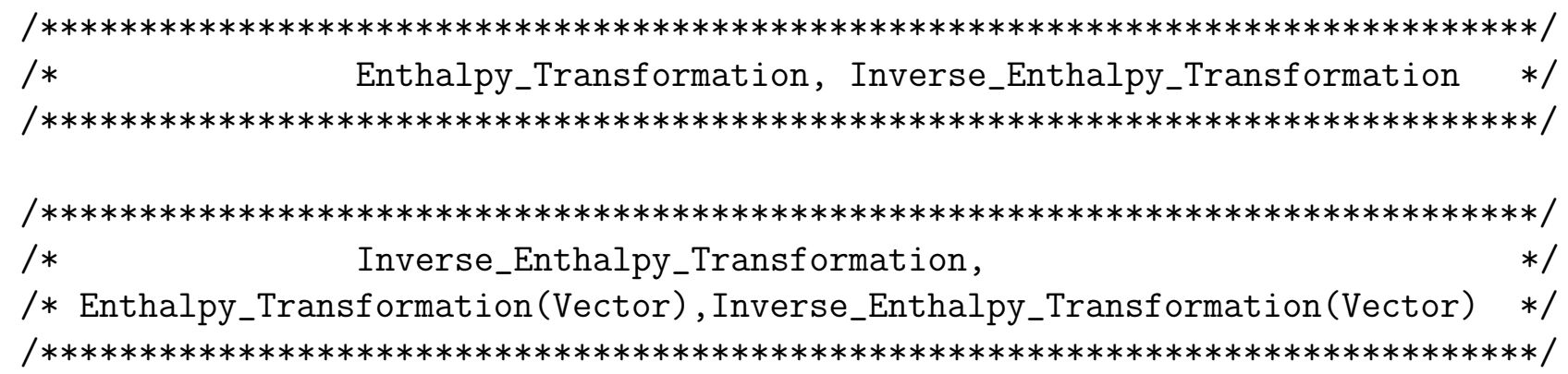

REAL EnthalpyTransformVal (const REAL T_val)

\{

FUNCNAME ("EnthalpyTransformVal");

REAL H_val ;

REAL TL, TR ;

REAL c_reg ;

$\mathrm{TL}=\mathrm{T}$ _melting - epsReg $; \mathrm{TR}=\mathrm{T}$ _melting + epsReg ;

c_reg $=0.5 *\left(\left(c_{-} s * r h o_{-} s+c_{-} l * r h o_{-} l\right)+\right.$ rho_s $*$ Latent/epsReg $)$;

if (T_val<=T_melting)

$\mathrm{H}_{-} \mathrm{val}=$ rho_s $* c_{-} \mathrm{s} * \mathrm{~T}_{-} \mathrm{val}+$ rho_s*0.5*Latent*exp (-fabs (T_val - T_melting)/epsReg);

else

H_val = rho_s*c_s $* T_{-}$melting + rho_l*c_l*(T_val - T_melting $)+$ rho_s*0.5*Latent*( $1.0-\exp \left(-\mathrm{fabs}\left(\mathrm{T}_{-}\right.\right.$val - T_melting)/epsReg $\left.)\right)$;

\}

$\operatorname{return}\left(\mathrm{H}_{-} \mathrm{val}\right)$; 
REAL InverseEnthalpyTransformVal (const REAL H_val)

\{

FUNCNAME ("InverseEnthalpyTransformVal");

REAL T_val;

REAL rhoCeS, rhoCeL ;

REAL TL, TR, HL, HR ; REAL c_reg ;

rhoCeS $=$ rho_s $s C_{-} \mathrm{S} ; \quad$ rhoCeL $=$ rho_l $1 * c_{-} 1 ;$

$\mathrm{TL}=\mathrm{T}$ _melting - epsReg $; \mathrm{TR}=\mathrm{T}$ _melting + epsReg ;

c_reg $=0.5 *\left(\left(c_{-}\right.\right.$s $*$ rho_s $\left.+c_{-} 1 * r h o_{-} 1\right)+$ rho_s $*$ Latent/epsReg $)$;

$\mathrm{HL}=\operatorname{rhoCeS} * \mathrm{TL} ; \quad \mathrm{HR}=\operatorname{rhoCeS} * \mathrm{TL}+\mathrm{c}_{-} r$ reg* $(\mathrm{TR}-\mathrm{TL}) ;$

if $\left(\mathrm{H}_{-} \mathrm{val}<\mathrm{HL}\right)$

T_val = H_val/rhoCeS ; $*$ value of IET

else

\{

if $\left(\mathrm{HL}<\mathrm{H}_{-} \mathrm{val}<\mathrm{HR}\right)$

$T_{-} v a l=\left(H_{-} v a l-r h o_{-} s * C_{-} s * T L\right) / c_{-} r e g+T L ;$ else

T_val $=\left(H_{-}\right.$val - rho_s*C_s*TL $\left.-c_{-} r e g *(T R-T L)\right) /$ rhoCeL ; \}

\}

$\operatorname{return}\left(T_{-} v a l\right) ;$

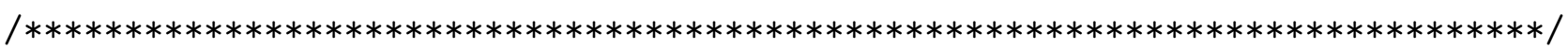

/* Kirchoff_Transformation, Inverse_Kirchoff_Transformation */

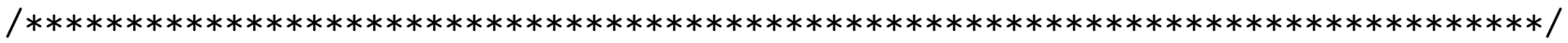

REAL Heat_conductivity(const REAL T)

\{

FUNCNAME("Heat_conductivity");

REAL k_val ; 


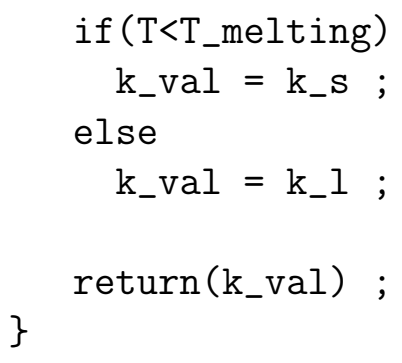




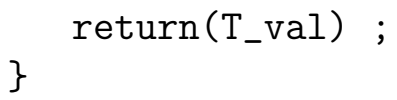




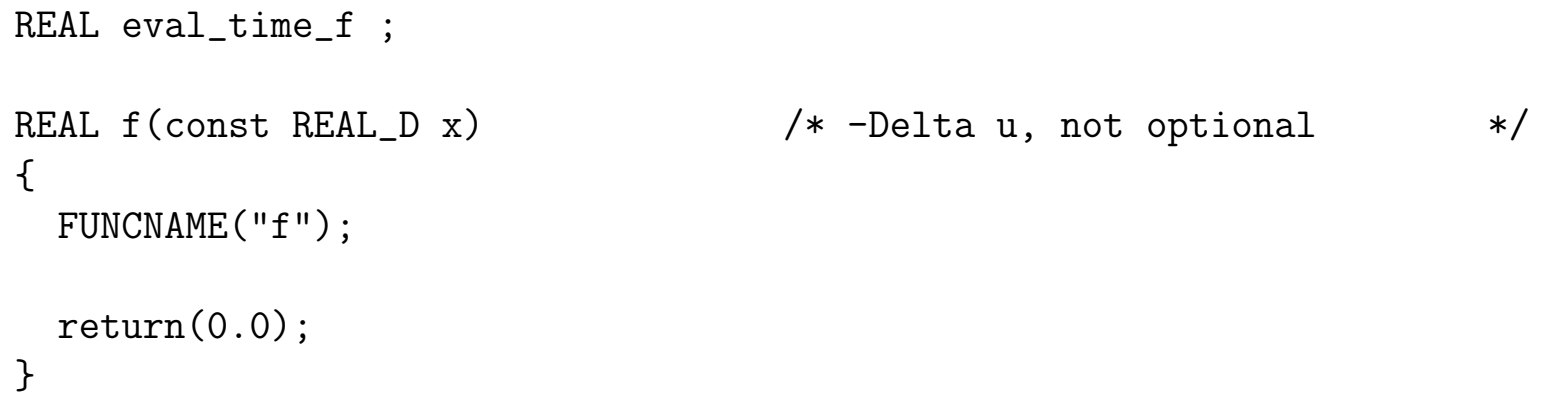




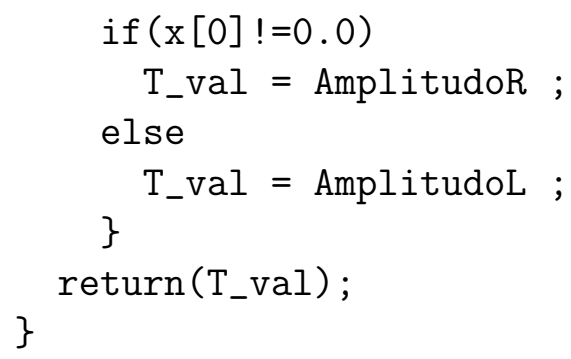

REAL eval_time_H ;

REAL eval_time_u_D ; 


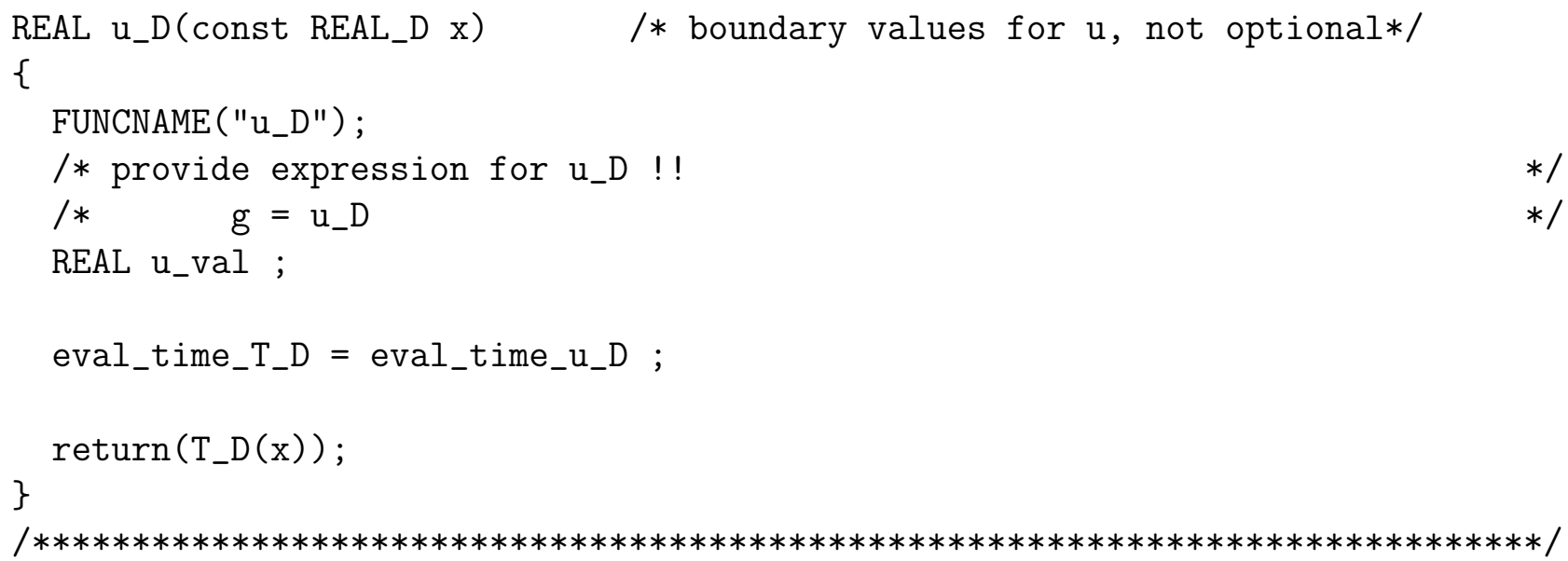

\section{Remark}

In principle, the main driver program able to cater temperature dependent material parameter. This pointed out previously through a user supplied functions, i.e. called by the driver program through UtoH function which called by nlsor in the solver(). Within current implementation, we dealt only constant properties of material. To extends it's capability, the extension needed is: implement efficiently the functions $\beta^{-1}(u)$, which is not available in general case. One way to handled this is through an array storing Temperature $(\Theta)$, Enthalpy $(H)$, and generalized Temperature $(u)$, as a (dynamic) table, equiped with an efficient search function on it. This requirement is important, since during each iteration in the nlsor, the functions called twice for each vector component being iterated. 


\section{APPENDIX B. Nonlinear SOR source Code.}

We give in the following a complete source code for nonlinear SOR solver, whereonly the function $r$ is available. In the case its derivative is available, the code is easily modified, by replacing numerical approximation of derivatives in the newton iteration of root finder by a supplied analytical derivatives. An example of the prototype routine of this case is of the following :

int nlsor_s (const DOF_MATRIX $* a$, const DOF_REAL_VEC $* b$, const DOF_REAL_VEC $* f$, const DOF_SCHAR_VEC *bound, REAL (*phi) (const REAL),

REAL ( $*$ Dphi) (const REAL), DOF_REAL_VEC $* u$, REAL omega, REAL tol, int max_iter, int info)

The complete nonlinear SOR, where the analytical derivative is not available are provided in the next code write-up.

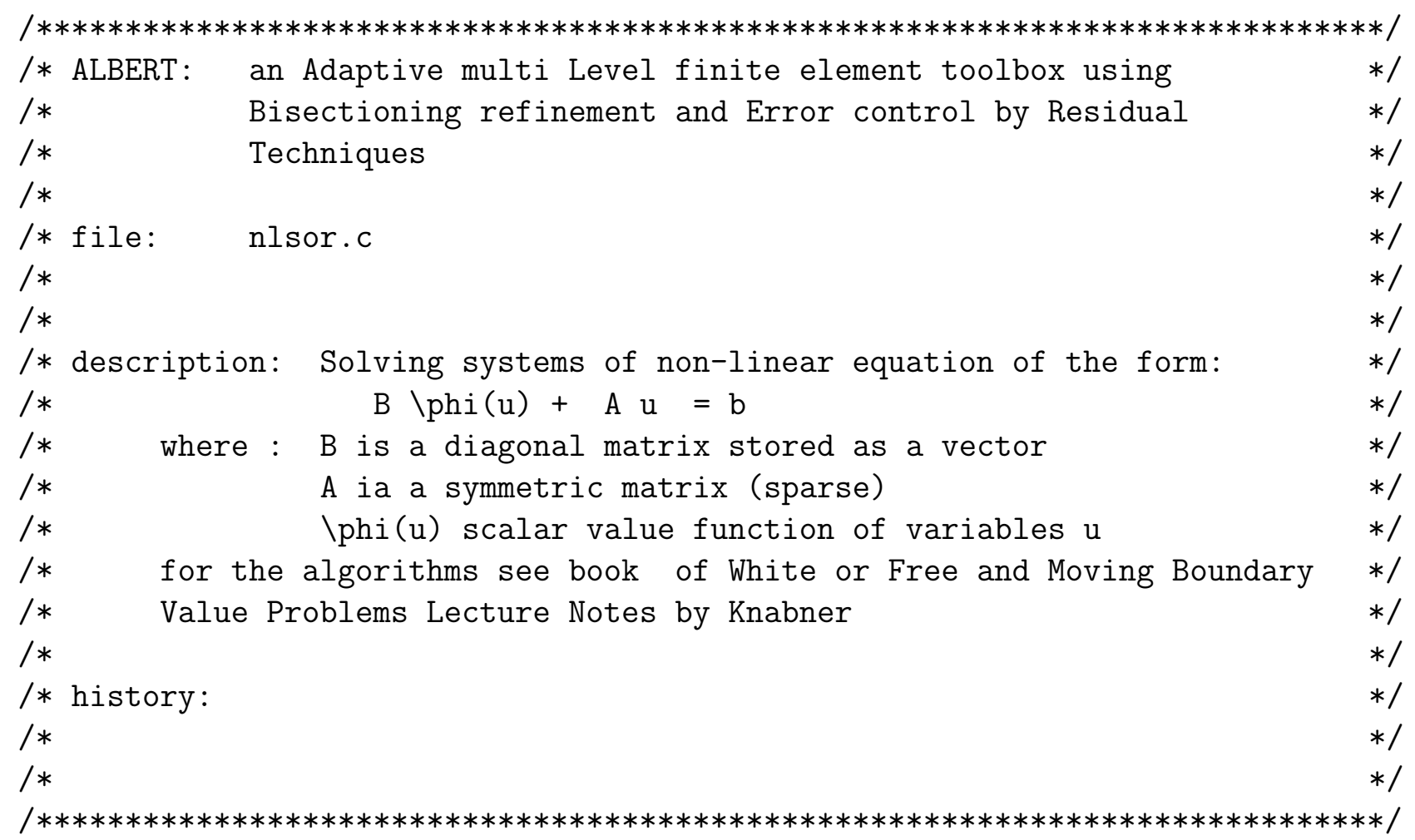




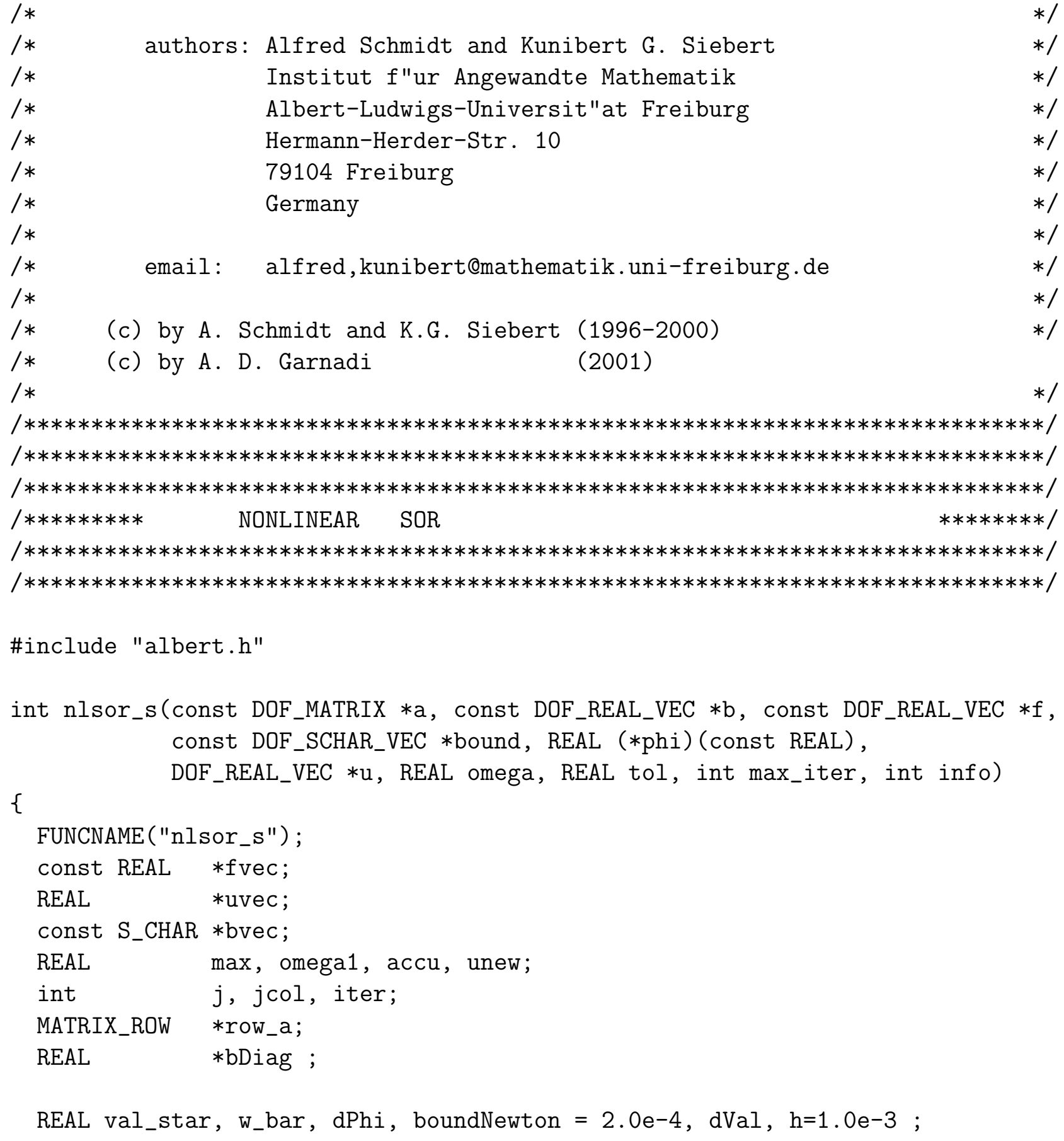

REAL val_star, w_bar, dPhi, boundNewton $=2.0 \mathrm{e}-4, \mathrm{dVal}, \mathrm{h}=1.0 \mathrm{e}-3$; 


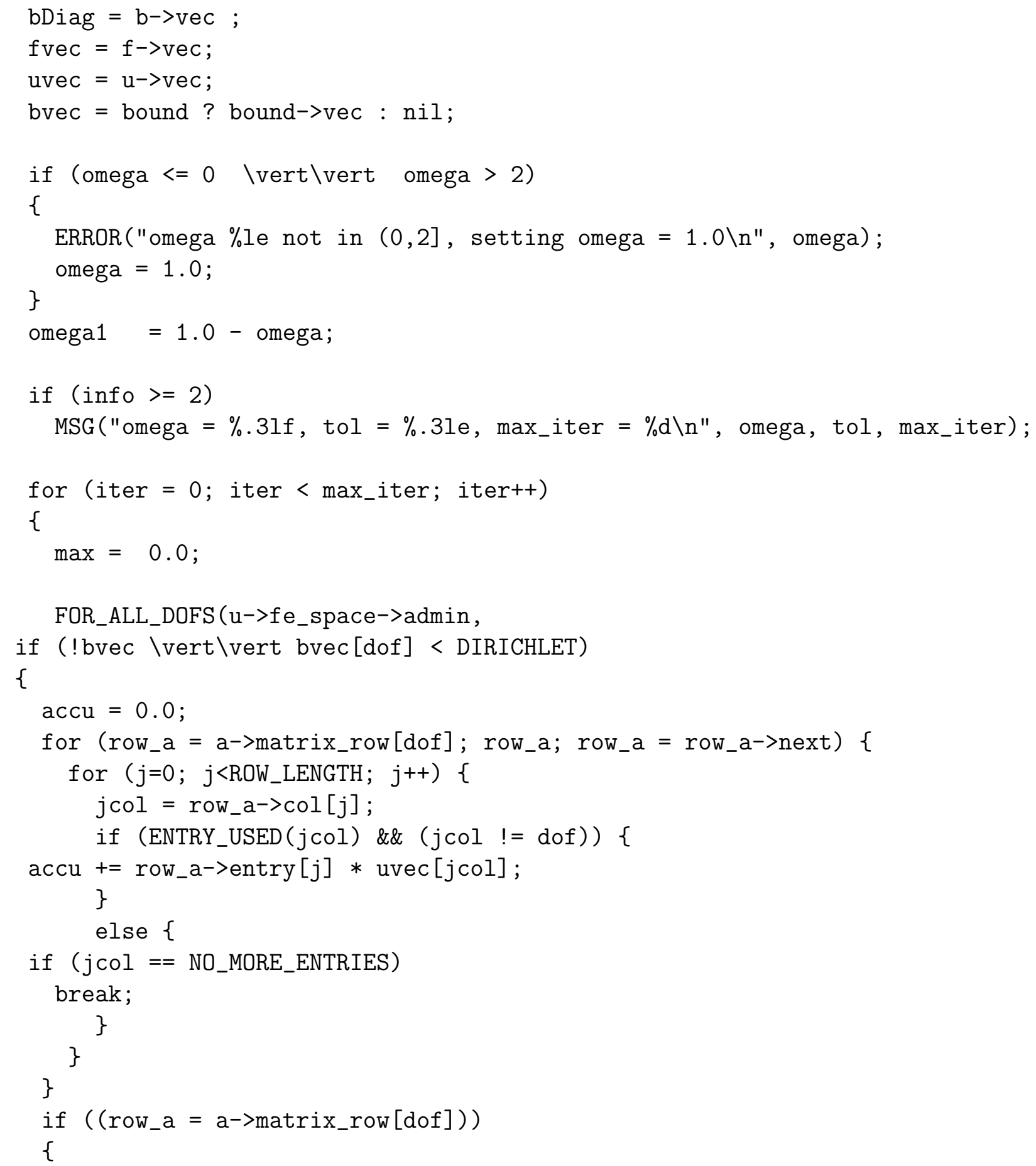




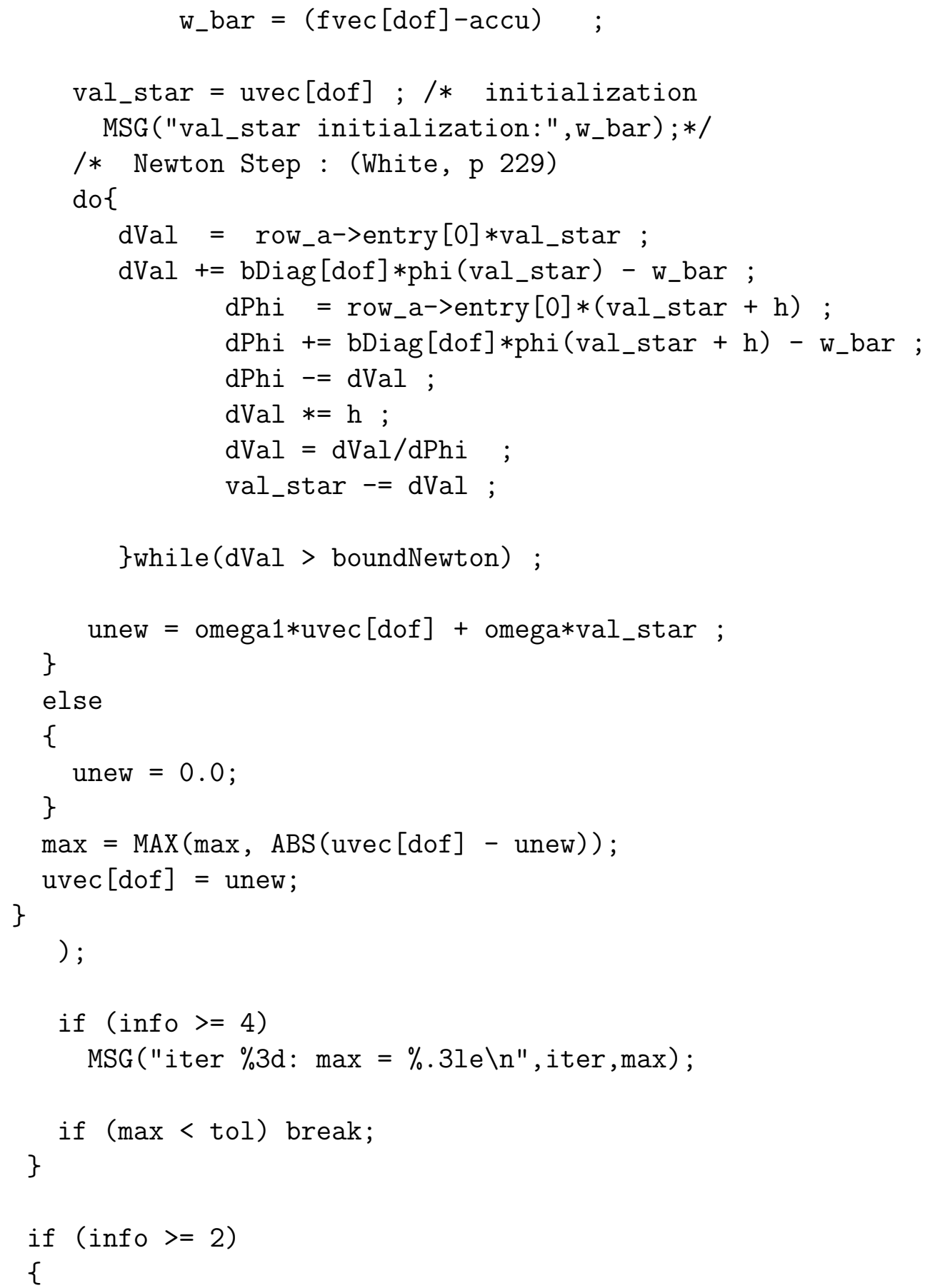




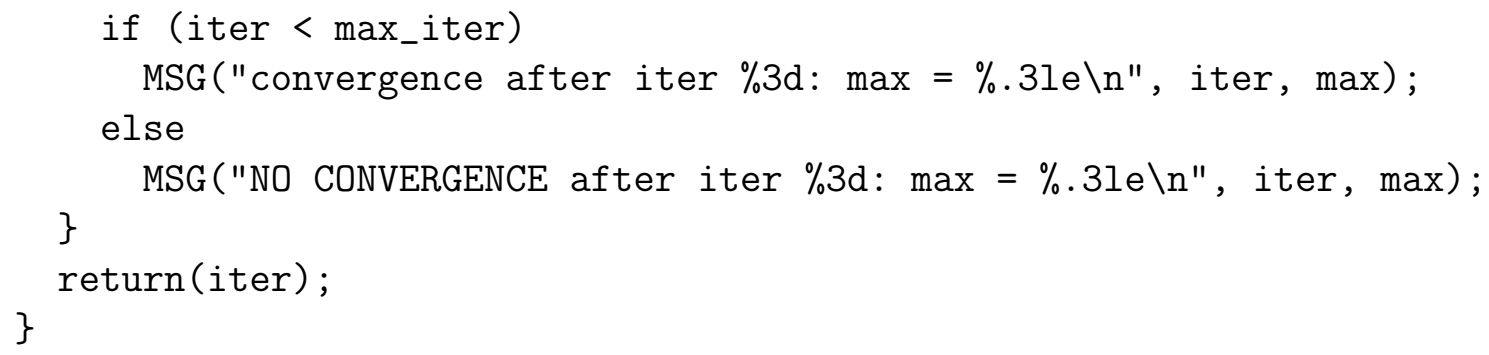

